Nota científica

(Short communication)

\title{
INCIDENCIA DEL PARASITOIDE CALLIEPHIALTES GRAPHOLITHAE CRESSON (HYMENOPTERA: ICHNEUMONIDAE) EN LARVAS DE CYDIA CARYANA FITCH (LEPIDOPTERA: TORTRICIDAE)
}

\section{ASSOCIATION BETWEEN THE PARASITOD CALLIEPHIALTES GRAPHOLITHAE CRESSON (HYMENOPTERA: ICHNEUMONIDAE) AND LARVAE OF CYDIA CARYANA FITCH (LEPIDOPTERA: TORTRICIDAE)}

\author{
NÉSTOR BAUTISTA-MARTÍNEZ ${ }^{1}$, CARLOS PATRICIO ILLESCAS-RIQUELME ${ }^{1 *}$, JOSÉ ANTONIO RAMOS- \\ MARTÍNEZ ${ }^{2}$, ENRIQUE RUIZ-CANCINO ${ }^{3}$ \\ ${ }^{1}$ Programa Entomología y Acarología, Colegio de Postgraduados, Campus Montecillo. Montecillo, Texcoco, CP 56230, Estado de \\ México, México.<nestor@colpos.mx> \\ ${ }^{2}$ Comité Estatal de Sanidad Vegetal de Guanajuato. Av. Siglo XXI No. 1156 Predio Los Sauces, CP 36547, Irapuato, Guanajuato, \\ México \\ ${ }^{3}$ Facultad de Ingeniería y Ciencias, Universidad Autónoma de Tamaulipas, Centro Universitario CP 87149, Cd. Victoria, \\ Tamaulipas, México.<eruiz@docentes.uat.edu.mx> \\ *Autor de correspondencia: <entoillescas@gmail.com>
}

Recibido: 27/02/2018; aceptado: 24/05/2018; publicado en línea: 30/10/2018

Editor responsable: Arturo Bonet

Bautista-Martínez, N., Illescas-Riquelme, C. P., Ramos-Martínez, J. A., Ruiz-Cancino E. (2018) Incidencia del parasitoide Calliephialtes grapholithae Cresson (Hymenoptera: Ichneumonidae) en larvas de Cydia caryana Fitch (Lepidoptera: Tortricidae). Acta Zoológica Mexicana (nueva serie.), 34, 1-4; https://doi.org/10.21829/azm.2018.3412124

RESUMEN. Se reporta la asociación entre el parasitoide Calliephialtes grapholithae y la larva de Cydia caryana. Se colectaron nueces pecaneras en el área de Jaumave, Tamaulipas, después las frutas fueron confinadas en cajas plásticas para esperar la emergencia de los adultos. Otro himenóptero que emergió durante el estudio fue Eupelmus sp.; sin embargo, su asociación no puede ser confirmada debido a que podría comportarse como parasitoide o hiperparasitoide.

Bautista-Martínez, N., Illescas-Riquelme, C. P., Ramos-Martínez, J. A., Ruiz-Cancino E. (2018) Association between the parasitod Calliephialtes grapholithae Cresson (Hymenoptera: Ichneumonidae) and larvae of Cydia Caryana Fitch (Lepidoptera: Tortricidae). Acta Zoológica Mexicana (nueva serie), 34, 1-4; https://doi.org/10.21829/azm.2018.3412124

\begin{abstract}
In the present work, the association between the parasitoid Calliephialtes grapholithae and larvae of Cydia caryana is reported. Pecan nuts collections were made in the area of Jaumave, Tamaulipas, later the fruits were confined in plastic containers to wait for the emergence of adults. Another hymenopteran that emerged in the study was Eupelmus sp., its association could not be confirmed since it can be conferred as a parasitoid or hyperparasitoid.
\end{abstract}


México ocupa el segundo lugar a nivel mundial en producción de nuez pecanera, Carya illinoensis (Wangenh.) K. Koch, se exporta a 19 destinos internacionales, incluyendo Canadá, Estados Unidos, Panamá, Colombia, España, Italia, Rusia, Vietnam, China, Hong Kong y Japón (SAGARPA, 2018).

En 2016 se registró un incremento del 86\% de la producción de nuez pecanera en relación al año anterior, alcanzando las 338,660 toneladas (SAGARPA, 2018). De acuerdo con el SIAP (2018) los principales estados productores de nuez pecanera en México son Chihuahua, Coahuila, Tamaulipas y Sonora.

Uno de los factores que limitan la producción de nuez pecanera es el impacto de las plagas. En México, los insectos que afectan a este cultivo son el gusano telarañero Hyphantria cunea Drury (Lepidoptera: Erebidae), los áfidos Monellia caryella Fitch, Monelliopsis pecanis Bissell y Melanocallis caryaefoliae Davis (Hemiptera: Aphididae), el gusano barrenador de la nuez Acrobasis nuxvorella Neunzig (Lepidoptera: Pyralidae) y el barrenador del ruezno Cydia caryana Fitch (Lepidoptera; Tortricidae) (Pinson-Domínguez \& Kasparyan, 2005; Tarango et al., 2013 a y b). De las especies antes mencionadas, las palomillas A. nuxvorella y C. caryana son las más relevantes al mermar directamente la producción.

A pesar de ser un cultivo de interés económico, los trabajos en relación a la fitosanidad y a la entomofauna asociada al nogal pecanero son limitados. En este sentido, es necesario conocer los enemigos naturales de estas plagas para la toma de decisiones relacionada con el manejo.

Para el presente trabajo, se visitaron huertos productores de nogal pecanero pertenecientes al municipio de Jaumave, Tamaulipas, México. Generalmente en este lugar existen importantes pérdidas en la producción debido a la incidencia de barrenadores del ruezno.

El día 05 de diciembre de 2015 se colectaron un total de $15.62 \mathrm{~kg}$ de nuez pecanera de 7 árboles escogidos de manera aleatoria. Las nueces se confinaron en recipientes de plástico (base de $30 \mathrm{~cm}$ diámetro x $16.5 \mathrm{~cm}$ de altura) y se cubrieron con tela de organza para esperar la emergencia de insectos adultos. El material colectado se ingresó a la cámara de cría y permaneció en los contenedores a $25 \pm 3{ }^{\circ} \mathrm{C}$ con fotofase 12:12 durante 3 meses hasta el 18 de marzo de 2016. Del mismo modo, al momento de la colecta, se eligieron 50 nueces para abrirlas y contabilizar el número de larvas sanas y parasitadas. Después de revisarlas, las larvas se confinaron junto con el tejido vegetal en otro contenedor por separado para la emergencia de los adultos de la palomilla y/o sus parasitoides.

Con base en el conocimiento previo de que C. caryana es la única especie de este género que ataca la nuez pecanera en la zona de estudio, se utilizaron las claves de Gilligan \& Passoa (2014) para corroborar la identidad de las larvas de Cydia; adicionalmente se corroboró la identificación de adultos de C. caryana, utilizando las claves de Gilligan \& Epstein (2012). Por otro lado, los parasitoides adultos emergidos pertenecieron a la subfamilia Pimpilidae (Ichneumonidae), y se determinaron a nivel de especie con las claves de Townes \& Townes (1960).

Del total de insectos colectados nueve fueron el barrenador del ruezno, $C$. caryana y dos especies de parasitoides, Calliephialtes grapholithae (Hymenoptera: Ichneumonidae) ( $\mathrm{n}=21,17 \uparrow$ y $4{ }^{\Uparrow}$ ) (Fig. 1A) y Eupelmus sp. (Hymenoptera: Eupelmidae) (n=4, 3 P ) (Fig. 1B).

De las nueces estudiadas por separado $(\mathrm{n}=50)$, dieciocho tenían presencia de larvas del barrenador del ruezno, conteniendo de 1 a 5 por fruto con un porcentaje de parasitismo del 8\% ocasionado por $C$. grapholitae. El material se encuentra depositado en la colección del área de Entomología Agrícola de Colegio de Postgraduados, Campus Montecillo en Texcoco, Estado de México, México. 

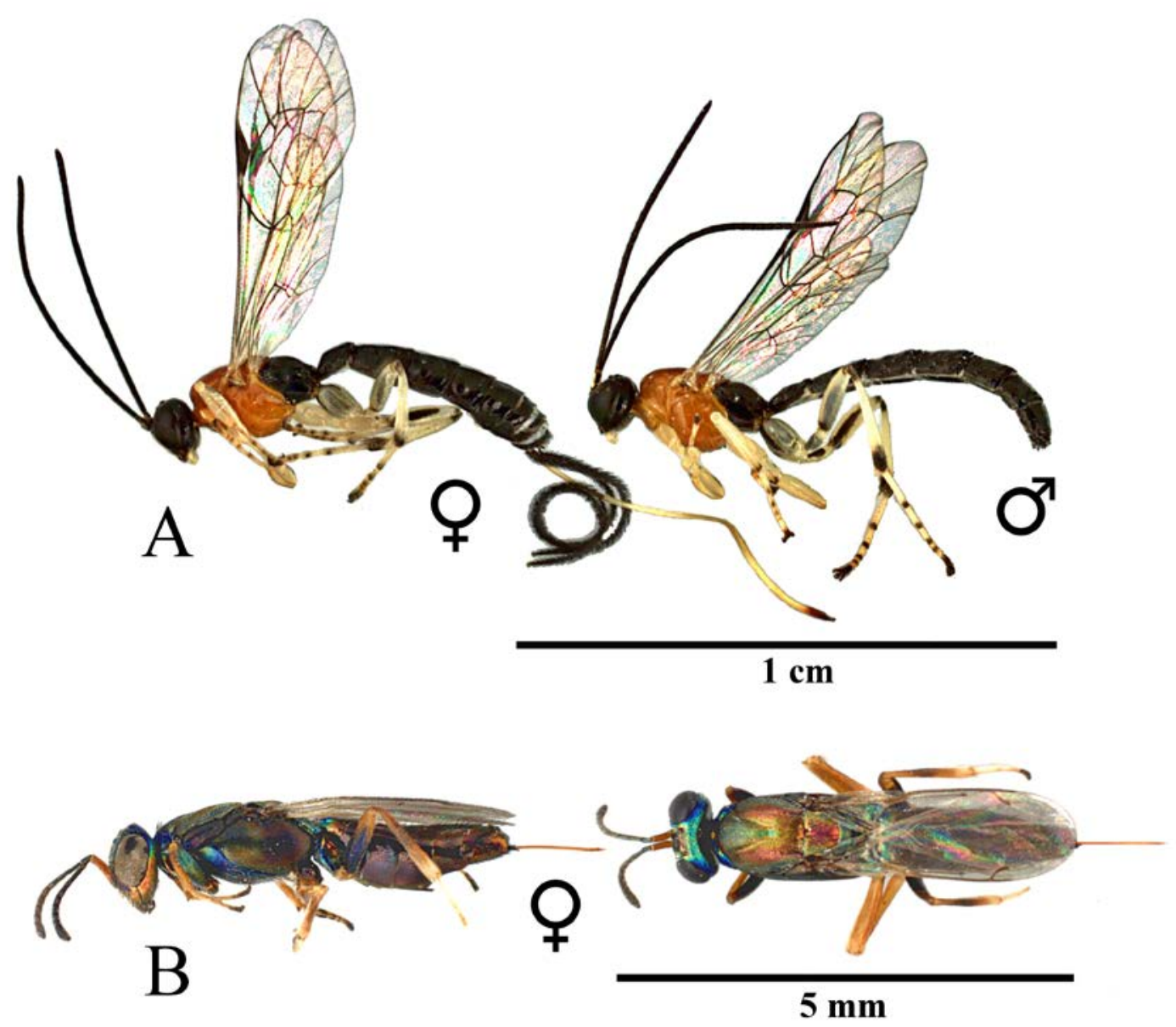

Figura 1. A) Calliephialtes grapholithae (ambos sexos) y B) Eupelmus sp. (hembra).

Calliephialtes grapholithae ya había sido reportada en México por Pinson-Domínguez \& Kasparyan (2005) en la misma zona productora de nuez pecanera de Jaumave, Tamaulipas, sin embargo, no se señala una asociación directa de este parasitoide con las larvas de $C$. caryana. Lo anterior comúnmente sucede cuando sólo se colectan adultos y se tiene más de un hospedante posible, por lo tanto la relación parasitoide - hospedero no estaba confirmada.

En el presente estudio no se encontró al barrenador de la nuez A. nuxvorella aunque si se conoce su presencia en la zona de estudio, es por ello que se sugiere una relación directa de $C$. grapholithae con las larvas de C. caryana, ya que además existen otros reportes de este parasitoide atacando a C. caryana en el estado de Texas, Estados Unidos (Gunasena \& Harris, 1988).

El segundo parasitoide obtenido fue Eupelmus sp., el cual no fue identificado a nivel de especie por no existir claves de este género para México. Sin embargo, Pinson-Domínguez \& Kasparyan (2005) mencionan a Eupelmus sp., parasitando barrenadores del ruezno en la misma zona de estudio y ReyesVillanueva (1987) reporta a Eupelmus sp., Eupelmus cyaniceps Ashmed y Eupelmus limneridae Howard en larvas de C. caryana en el estado de Nuevo León. Del mismo modo, Gunasena \& Harris (1988) reportaron a E. amicus y E. limneriae parasitando a A. nuxvorella en Texas; pero mencionan a E. amicus como un hiperparasitoide polífago que incluso ataca larvas de C. grapholithae. Con base en lo anterior no 
es posible determinar si la especie de Eupelmus encontrada es parasitoide o hiperparasitoide sobre $C$. caryana.

\section{LITERATURA CITADA}

Finlayson, T. (1967). Taxonomy of final-instar larvae of the hymenopterous and dipterous parasites of Acrobasis spp. (Lepidoptera: Phycitidae) in the Ottawa region. The Canadian Entomologist, 99, 1233-1271.

Gilligan, T. M. \& Epstein M. E. (2012). TortAI, Tortricids of Agricultural Importance to the United States (Lepidoptera: Tortricidae). Identification Technology Program (ITP), USDA/APHIS/PPQ/CPHST, Fort Collins, CO. Available at: <http://idtools.org/id/leps/tortai/> (accessed on December 2017.

Gilligan, T. M. \& Passoa S. C. (2014). LepIntercept, An identification resource for intercepted Lepidoptera larvae. Identification Technology Program (ITP), USDA/APHIS/PPQ/S\&T, Fort Collins, CO. Available at: <www.lepintercept.org> (accessed on January 2018).

Gunasena, H. S. \& Harris M. K. (1988). Parasites of hickory shuckworm and pecan nut casebearer with five new host-parasites records. Southwestern Entomologist, 13, 107-111.

Pinson-Domínguez, O., J. F. \& Kasparyan, D. R. (2005). Registro de Calliephialtes grapholithae (Cresson) (Hymenoptera: Ichneumonidae) parasitando lepidópteros plaga en rueznos de nogal en Jaumave, Tamaulipas, México. Pp 25-28. En: XXVII Congreso Nacional de Control Biológico, San Miguel de Allende, Gto., México.

Reyes-Villanueva., F. (1987). Insectos parásitos de los lepidópteros plaga del nogal en Nuevo León; análisis de su potencialidad como agentes de control biológico. Folia Entomológica Mexicana, 72,111-120.

SAGARPA. (2018). Secretaría de Agricultura, Ganadería, Desarrollo Rural, Pesca y Alimentación. $<$ http://www.sagarpa.gob.mx/Delegaciones/chihuahua/boletines/Paginas/B003-CSCH012017.aspx> (Fecha de consulta: 13 Feb 2018)

SIAP. (2018). Nuez; Cierre de la producción agrícola. Anuario Estadístico de la Producción Agrícola datos 2016. <http://nube.siap.gob.mx/cierre_agricola> (Consultado el 13 Feb 2018).

Tarango R. S. H., García N. G. \& Burrola M. J. R. (2013a). Manejo de áfidos del nogal pecanero. Instituto Nacional de Investigaciones Forestales, Agrícolas y Pecuarias; Delicias Chihuahua. No 33. 2 Ed. 43 p.

Tarango R. S. H., García N. G. \& Burrola M. J. R. (2013b). Manejo del Barrenador del Ruezno en Chihuahua. Instituto Nacional de Investigaciones Forestales, Agrícolas y Pecuarias; Delicias Chihuahua. No. 42.36 p.

Townes, H. \& Townes, M. (1960). Ichneumon-Flies of America North of Mexico. 2. Subfamilies Ephialtinae, Xoridae, Acaenitinae. U. S. National Museum. Bulletin 2016, Part 2. 676 pp. 